This is a repository copy of Guaranteed estimation and distributed control of vehicle formations" in the Depósito de Investigación de la Universidad de Sevilla

Version: Author Accepted Version

Citation: R. A. García, L. Orihuela, P. Millán, F. R. Rubio \& M. G. Ortega. "Guaranteed estimation and distributed control of vehicle formations". International Journal of Control - pp. 1-15. Enero 2020. 10.1080/00207179.2020.1714074

To cite this publication, please use the final published version (if applicable). Please check the document version above.

Copyright: Other than for strictly personal use, it is not permitted to download, forward or distribute the text or part of it, without the consent of the author(s) and/or copyright holder(s), unless the work is under an open content license such as Creative Commons.

Takedown policy: Please contact us (idus@us.es) and provide details if you believe this document breaches copyrights. We will remove access to the work immediately and investigate your claim 


\title{
INTERVAL ESTIMATION
}

\section{Guaranteed Estimation and Distributed Control of Vehicle Formations}

\author{
Ramón.A. García ${ }^{a}$, L. Orihuelab ${ }^{b}$ P. Millán ${ }^{b}$, F.R. Rubio ${ }^{a}$ and M.G. Ortega ${ }^{a}$ \\ ${ }^{a}$ Dpt. Ingeniería de Sistemas y Automática, Universidad de Sevilla, Seville, Spain; ${ }^{\text {b}}$ Dpt. \\ Ingeniería, Universidad Loyola Andalucía, calle Energía solar, s/n, Sevilla, Spain
}

\begin{abstract}
ARTICLE HISTORY
Compiled January 8, 2020

ABSTRACT

This paper presents a distributed set-membership estimation and formation control algorithm for a fleet of vehicles. Each vehicle is equipped with an agent with sensing, acting, computational and communication capacities. The agent's objective is to compute estimation set for the state of all the fleet of vehicles and, additionally, generate control actions to keep its local vehicle in formation. In order to improve the estimation, the agents exchange information with other neighbouring agents. The estimation sets are mathematically characterized by zonotopes. The novelty of the proposed solution lies in the fact that the estimation is guaranteed in spite of the lack of knowledge of the control signal applied by the rest of vehicles. Then, in presence of bounded disturbances and noises, the position reference for the vehicles can be tracked with a bounded estimation. The proposed solution is tested by simulations.
\end{abstract}

\section{KEYWORDS}

Distributed estimation and control, fleet of vehicles, guaranteed estimation, zonotopes.

\section{Introduction}

The emergence of Wireless Sensor Networks (WSNs), together with the cheapening of IoT (Internet of Things) devices, has multiplied the number of tasks that can be performed in a distributed environment. Among them, distributed monitoring using a WSN is perhaps the field that has grown more in the last years. It is easy to find applications of distributed monitoring in many fields, such as agriculture (Bencini, Collodi, Di Palma, Manes, and Manes (2009)), water distribution system (M. Lin, Wu, and Wassell (2008)), pipelines (Stoianov, Nachman, and Madden (2007)), electrical power grids (Grilo et al. (2012)), structural health monitoring (Yu-liang, Yu, Lian-fen, Jian, and Ying (2012)), or traffic control systems (Tubaishat, Shang, and Shi (2007)), just to name a few.

It is important to mention that distributed estimation and distributed monitoring are not exactly the same, but sometimes these terms are confused with each other. Whereas distributed monitoring implies the deployment of a WSN to measure certain parameters, and to reduce uncertainty and increase robustness, distributed estimation additionally implies to get to know certain hidden variables that are not directly 
measured and that can be inferred from the data using a dynamical description of the plant.

The literature of distributed estimation is vast, since it has become a hot topic in the last years. Most of the estimation algorithms are modifications of classical centralized estimators to cope with the new distributed paradigm. Then, different modifications of the distributed Kalman filter have been proposed for plants affected by Gaussian noises and disturbances (Battistelli and Chisci (2016); Cattivelli and Sayed (2010); Das and Moura (2015); del Nozal, Orihuela, and Millán (2017); Olfati-Saber (2007); G. Wang, Li, and Zhang (2017)). Distributed Luenberger observers have been also developed for linear systems (del Nozal, Millán, Orihuela, Seuret, and Zaccarian (2019); Matei and Baras (2012); Mitra and Sundaram (2018)). For nonlinear systems, distributed Bayesian estimators have been developed in Forti et al. (2018). A complete survey of distributed estimation algorithms based on consensus strategies can be found in Garin and Schenato (2010).

However, the objective of guaranteed estimators is somewhat different. The estimation goal is to compute a set in which the actual state of the plant is contained for sure. Then, in addition to the estimation of the state, a guaranteed or set-membership estimator must provide a set that contains, with probability equal to one, the state of the system. In contrast to Kalman or stochastic filters, guaranteed estimators do not require to know the statistical description of noises and disturbances, but only their extreme bounds. Compared with classical methodologies, guaranteed observers finds their place in applications where the uncertainty in the estimation might become a critical issue, such as constrained control.

Perhaps the first feature that makes the difference between the approaches found in the literature is the mathematical description of the sets, being the most common solutions those based in ellipsoids (Wei, Liu, Song, and Liu (2015)), zonotopes (Alamo, Bravo, and Camacho (2005); Combastel (2015)) and interval observers (Chabane, Maniu, Alamo, Camacho, and Dumur (2014); Mazenc and Bernard (2011)). Recently, the combination of probability-based and set-membership estimation has lead to interesting novel formulations, as in Combastel (2016). Guaranteed estimators must not be confused with robust estimators, that deals with uncertain models of the plant dynamics, as in H. Dong, Wang, Alsaadi, and Ahmad (2015).

When it comes to the extension of the aforementioned formulation to a distributed paradigm, a revision of the state of the art reveals that the literature is rather scarce. Using a zonotope-based description, the authors in García, Rubio, Orihuela, Millán, and Ortega (2017); Keiffer (2009) were able to present distributed estimation algorithms that make use of intersections between sets. Another idea was used in Orihuela, Millán, Roshany-Yamchi, and Garcia (2018); Orihuela, Roshany-Yamchi, Garcia, and Millán (2017), where the authors presented a Kalman-inspired set-membership observer extending the idea in Combastel (2015) to a distributed framework with network-based problems. The natural extension of the previously mentioned work (Combastel (2016)) have been recently published in Combastel and Zolghadri (2018). In Y. Wang, Alamo, Puig, and Cembrano (2018) the authors propose a distributed zonotope-based estimator valid for a family of subsystems coupled by the state. Finally, the use of consensus techniques for distributed estimation with ellipsoidal sets was reported in Ma, Wang, Lam, and Kyriakoulis (2017).

Sometimes, the result of distributed estimation algorithms are used as inputs to distributed controllers. These are the so-called distributed joint estimation and control problem. When an agent is able to apply control actions to the plant, the estimation of the rest of agents is compromised, since those actions are unknown for the rest of 
agents. Hence, the solution for the joint problem is rather more complex. There exists a common line of research that proposes different formulations of a cascade structure, in which the estimation error must converge faster than the tracking error, see Rubio, Millán, Orihuela, and Vivas (2014); Zhang, Hengster-Movric, Sebek, Desmet, and Faria (2017). In all these papers, the authors assume that the control actions applied to the system at any moment are the same that the local agent would apply if it has access to all control channels. This incurs in an estimation error that disappears when the estimated states of every agent actually converge to the system state.

However, this idea cannot be applied directly to set-membership paradigms, since the described assumption will lead to non-guaranteed estimations in the transient. From the authors' knowledge there exists no paper in which this problem has been solved, even for linear systems. The only solutions are restricted to formulations of the plant divided into subsystems for which the estimation objectives are just local, as in Combastel and Zolghadri (2018); Orihuela et al. (2018, 2017); Y. Wang et al. (2018).

Distributed control methodologies have found application in the control of vehicle formations, where different approaches can be found in the literature either for path planning and formation control. For example, in Achtelik, Lynen, Weiss, Chli, and Siegwart (2014); Ousingsawat and Campbell (2004), path planning strategies are presented for aerial vehicles, ensuring knowledge of the intervals containing critical variables such as temperature, velocity, flow and so on. For surface vehicles, Liu and Bucknall (2015) tested its results in real navigation maritime environment, by using fast matching methodology to improve the path planning algorithm.

Regarding formation control, in Yang, Naeem, Irwin, and Li (2014) the task is transformed into a group of decentralized problems and a stability analysis is presented. In R. Dong and Geng (2016) a distributed control law is developed to achieve trajectory tracking for $N$ vehicles in rigid formation. For one-dimensional formation of vehicles, F. Lin, Fardad, and Jovanović (2012) compute optimal control based on the information of the nearest neighbours. More flexibility in the communication is taken into consideration in Peng, Wen, Rahmani, and Yu (2015), where the formation control problem is solved by a consensus algorithm, or in Millan, Orihuela, Jurado, and Rodriguez Rubio (2014), where the control strategy proposed makes possible to deal with delays and packet dropouts. Non-linear approaches found in Cai and De Queiroz (2015); Li, Shi, and Yan (2016) deal with backstepping and receding horizon control, respectively. For constrained underactuated underwater vehicles, the leader-follower paradigm has been successfully applied in Li, Xie, and Yan (2016). Application on a quadrotor platform is presented in X. Dong, Yu, Shi, and Zhong (2015). Finally, model and environmental uncertainties are considered in Peng, Wang, Chen, Hu, and Lan (2013); Xue, Wu, and Wang (2015), respectively.

This paper tackles the joint problem of distributed set-membership estimation and formation control for fleets of vehicles. The main contributions of the paper are:

- A distributed set-membership estimator is applied for the first time for a fleet of vehicles. It is able to estimate, in a guaranteed way, the position and velocity of each vehicle in a distributed way, assuming that the vehicles measure only local information. In particular, one vehicle, the leader, is able to measure its absolute position, while the others measure their relative position with respect to their neighbours. In the literature, we can find an application of set-membership estimation for the localization of a unique mobile robot in Zhou, Qian, Fang, Ma, and Dai (2015).

- The proposed estimator provides guaranteed sets in spite of the presence of 
unknown control actions that are being applied to other vehicles. Distributed set-membership estimators dealing with unknown control signals have been only successfully applied when the estimation objectives were local Combastel and Zolghadri (2018); Orihuela et al. (2018, 2017); Y. Wang et al. (2018).

In addition, the proposed methodology have some other relevant positive features. Firstly, the sets are characterized as zonotopes, this easing the transmission of information through the network. Secondly, both the estimator and the controller are designed in a decentralized way. Finally, although the stability is proven for vehicles with linear models, it is shown that the proposed algorithm can be applied to the unicycle model under some mild assumptions.

The paper is organized as follows. Section 2 presents the notation and preliminary concepts related to set-membership estimation. Section 3 describes the fleet of vehicles, the agents and formulates the problem under consideration. Section 4 presents the distributed estimation algorithm. The formation control is described in Section 5. The proposed solution is tested by simulations in Section 6. Finally, some conclusions and remarks are drawn in Section 7.

\section{Notation and Preliminaries}

A zonotope is an affine transformation of an unitary hypercube that can be used to characterize sets in a given state space. It will be represented with a calligraphic letter $\mathcal{X}$, and is defined by its centre $c \in \mathbb{R}^{n}$ and its generator matrix $H \in \mathbb{R}^{n \times b}$, that is:

$$
\mathcal{X}=\langle c, H\rangle \triangleq\left\{c+\sum_{i=1}^{b} \zeta_{i} h_{i}:\left|\zeta_{i}\right| \leq 1\right\},
$$

where $h_{i}$ represent a column of matrix $H$. The dimension of the zonotope is $n$ and its order is $b$. Let $\mathcal{X}=\left\langle c_{x}, H_{x}\right\rangle$ and $\mathcal{Y}=\left\langle c_{y}, H_{y}\right\rangle$ be two zonotopes, and $R$ a matrix, all of them of appropriate dimensions. Then, it holds:

$$
\begin{aligned}
R \mathcal{X} & =\langle R c, R H\rangle, \\
\mathcal{X} \oplus \mathcal{Y} & =\left\langle c_{x}+c_{y},\left[H_{x} H_{y}\right]\right\rangle,
\end{aligned}
$$

where $\oplus$ denotes the Minkowski sum of two sets.

The operator $\operatorname{red}(\cdot)$ consists in an order reduction of a zonotope, in such a way that the order of $\operatorname{red}(\mathcal{X})$ is smaller than the order of $\mathcal{X}$, and $\mathcal{X} \subseteq \operatorname{red}(\mathcal{X})$. The order of $\operatorname{red}(\mathcal{X})$ is $q$. This operation has been defined in the literature in different ways. Throughout this document, the order reduction will be defined as in Combastel (2015). According to that paper, if the order is to be reduced from $p$ to $q$, the procedure starts by sorting the columns of $H$ on decreasing vector norm to construct matrix $\bar{H}$ :

$$
\bar{H}=\left[\begin{array}{lllll}
h_{1} & \cdots & h_{j} & \cdots & h_{b}
\end{array}\right],\left\|h_{j}\right\|^{2} \geq\left\|h_{j+1}\right\|^{2},
$$

and enclosing the set $H_{<}$generated by the $b-q+n$ smaller columns into a box:

$$
\operatorname{red}(\langle c, H\rangle)= \begin{cases}\langle c, H\rangle, & b \leq q, \\ \left\langle c,\left[H_{>} \text {box }\left(H_{<}\right)\right]\right\rangle, & b>q,\end{cases}
$$


where

$$
\begin{aligned}
& H_{>}=\left[\begin{array}{lll}
h_{1} & \cdots & h_{q-n}
\end{array}\right], \\
& H_{<}=\left[\begin{array}{lll}
h_{q-n+1} & \cdots & h_{b}
\end{array}\right],
\end{aligned}
$$

and $\operatorname{box}(H) \in \mathbb{R}^{n \times n}$ is computed as

$$
\operatorname{box}(H)=\left[\begin{array}{ccc}
\sum_{j=1}^{b}\left|h_{1 j}\right| & \ldots & 0 \\
\vdots & \ddots & \vdots \\
0 & \ldots & \sum_{j=1}^{b}\left|h_{p j}\right|
\end{array}\right] .
$$

A strip can be used to characterize a set of states consistent with a given scalar measurement. It will be represented with a capital letter $S$, and is defined as the set $S \triangleq\left\{x:\left|c^{T} x-d\right|<\sigma\right\}$, where $d, \sigma \in \mathbb{R}$ and $c, x \in \mathbb{R}^{n}$.

The set of states resulting after the intersection of a strip and a zonotope, i.e. $\mathcal{X} \cap S$, or after the intersection of two zonotopes, i.e. $\mathcal{X}_{1} \cap \mathcal{X}_{2}$, can be enclosed by a new zonotope. With some abuse of notation, we will refer this new zonotope $\mathcal{Z}$ as $\mathcal{Z} \triangleq \mathcal{X} \cap S$ or $\mathcal{Z} \triangleq \mathcal{X}_{1} \cap \mathcal{X}_{2}$. The construction of such zonotopes is well described in Alamo et al. (2005).

An estimation zonotope computed by agent $i$ at time instant $k$ with the information available at $k$ is denoted as $\hat{\mathcal{X}}_{i}(k \mid k)$. Furthermore, a prediction zonotope at instant $k+1$ with the information available at $k$ is denoted as $\hat{\mathcal{X}}(k+1 \mid k)$.

The connectivity of the agents is topologically defined by a graph $G=(V, E)$. The graph $G$ is assumed connected and undirected, with vertices $V=\{1,2, \ldots, p\}$ and edges $E \subset V \times V$. The set of vertices to which vertex $i$ is connected is named the neighbourhood of $i$ and is denoted by $\mathcal{N}_{i} \equiv\{j:(i, j) \in E\}$. Edge $(i, j)$ implies that vertex $i$ sends information to vertex $j$. The distance between vertex $i$ and vertex $j$, measured as the number of edges in a shortest path connecting them, is $d_{i j}$. Finally, the diameter of the graph $\bar{d} \triangleq \max \left\{d_{i j}\right\}$ is the greatest distance between any pair of vertices.

\section{Problem formulation}

This paper presents some contributions to the problem of estimation and formation control of fleet of vehicles. The objective is to present a distributed control algorithm that can be implemented when the disturbances affecting the vehicles are unknown but bounded. The distributed controllers require to know the state of the vehicles, which is not directly measurable. In order to solve this problem, a distributed guaranteed estimator is presented, in such a way that it is able to deal with the possible measurement noises and, at the same time, incorpore the information received from other estimators. The solution for the joint problem of guaranteed estimation and control and the complete algorithm that will be implemented in every agent is given in Sections 4-5.

Prior to that, the description of the fleet of vehicles and of the agents are given in the next subsections. The control and estimation problem will be formally stated at the end of this section. 


\subsection{Fleet description}

We consider a fleet of vehicles characterized as in Lafferriere, Williams, Caughman, and Veerman (2005), where $p$ vehicles with the same dynamics ${ }^{1}$ are considered:

$$
\dot{x}_{i}(t)=A_{v e h}^{c} x_{i}(t)+B_{v e h}^{c} u_{i}(t), \quad i=1 \ldots p,
$$

where the entries of $x_{i} \in \mathbb{R}^{2 r}$ represent $r$ configuration variables for vehicle $i$ and their derivatives, and $u_{i} \in \mathbb{R}^{r}$ represents control inputs. As in Lafferriere et al. (2005), it is further assumed that matrices $A_{v e h}^{c}$ and $B_{v e h}^{c}$ have the form:

$$
A_{v e h}^{c}=\operatorname{diag}\left\{\left[\begin{array}{cc}
0 & 1 \\
a_{21}^{1} & a_{22}^{1}
\end{array}\right], \ldots,\left[\begin{array}{cc}
0 & 1 \\
a_{21}^{r} & a_{22}^{r}
\end{array}\right]\right\}, B_{v e h}^{c}=I_{r} \otimes\left[\begin{array}{l}
0 \\
1
\end{array}\right]
$$

where $\otimes$ stands for the Kronecker product of two matrices. This model corresponds to the situation in which the individual configuration variables are decoupled and the acceleration is controlled separately. The odd-numbered entries of $x_{i}$ represent position-like variables, whereas the even-numbered entries represent velocity-like variables.

The previous dynamics are discretized with sampling time $T_{s}$ and additive disturbances are included:

$$
x_{i}(k+1)=A_{v e h} x_{i}(k)+B_{v e h} u_{i}(k)+w_{i}(k), \quad i=1 \ldots p, \quad x_{i} \in \mathbb{R}^{2 r},
$$

where $A_{v e h}, B_{v e h}$ correspond to the discrete-time version of $A_{v e h}^{c}, B_{v e h}^{c}$. The disturbances $w_{i}(k) \in \mathbb{R}^{2 r}$ can be caused by various reasons, such as non-modelled motor dynamics, lack of accuracy in certain actuators, wind (aerial or ground vehicles), currents (water vehicles), etcetera. Throughout the paper, we consider that these disturbances are unknown, but they always belong to known bounded sets, that is, $w_{i}(k) \in \mathcal{W}_{i}$. These sets are modeled as:

$$
\mathcal{W}_{i}=\left\langle 0, Q_{i}\right\rangle
$$

Let $x(k), u(k), w(k)$ be defined by stacking all the state vectors, control actions and disturbances of each vehicle, that is, $x(k)=\left[\begin{array}{llll}x_{1}(k)^{T} & x_{2}(k)^{T} \ldots x_{p}(k)^{T}\end{array}\right]^{T}, u(k)=$ $\left[\begin{array}{lllll}u_{1}(k)^{T} & u_{2}(k)^{T} & \ldots & u_{p}(k)^{T}\end{array}\right]^{T}, w(k)=\left[\begin{array}{llll}w_{1}(k)^{T} & w_{2}(k)^{T} & \ldots & w_{p}(k)^{T}\end{array}\right]^{T}$. Then, the dynamics of the complete fleet can be written as follows:

$$
x(k+1)=A x(k)+B u(k)+w(k)
$$

where $A \triangleq I_{p} \otimes A_{v e h}$ and $B \triangleq I_{p} \otimes B_{v e h}$.

\subsubsection{Unicycle model for the vehicles}

The presented formulation of the vehicles as linear systems in (4), although simple, can be used for more complicated models. In particular, this section summarises the results in Lawton, Beard, and Young (2003), in which they present an output feedback

\footnotetext{
${ }^{1}$ The estimation and control algorithm presented in the next section is still valid if vehicles with different dynamics are considered. For the matter of simplicity in the notation, we consider the case of equivalent vehicles.
} 
linearizing control for the control of the hand position of a vehicle, whose dynamics can be written as a double integrator.

The hand position of the vehicle is a point $h_{i}=\left[h_{i}^{x}, h_{i}^{y}\right]$ that lies a distance $L_{i}$ along the line that is normal to the wheel axis and intersects the wheel axis at the center point $b_{i}=\left[b_{i}^{x}, b_{i}^{y}\right]$, as shown in Figure 1 . As it is well known, the dynamical equations of the unicycle model are:

$$
\left[\begin{array}{c}
\dot{b}_{i}^{x} \\
\dot{b}_{i}^{y} \\
\dot{\theta}_{i} \\
\dot{s}_{i} \\
\dot{\omega}_{i}
\end{array}\right]=\left[\begin{array}{c}
s_{i} \cos \left(\theta_{i}\right) \\
s_{i} \sin \left(\theta_{i}\right) \\
\omega_{i} \\
0 \\
0
\end{array}\right]+\left[\begin{array}{cc}
0 & 0 \\
0 & 0 \\
0 & 0 \\
\frac{1}{m_{i}} & 0 \\
0 & \frac{1}{I_{i}}
\end{array}\right]\left[\begin{array}{c}
F_{i} \\
\tau_{i}
\end{array}\right],
$$

where $b_{i}=\left[b_{i}^{x}, b_{i}^{y}\right]^{T}, \theta_{i}, s_{i}, \omega_{i}$ are, respectively, the inertial position, orientation, linear speed and angular speed of vehicle $i$. The mass and moment of inertia of the vehicle are $m_{i}$ and $I_{i}$. The input signals are the torque $\tau_{i}$ and the force $F_{i}$.

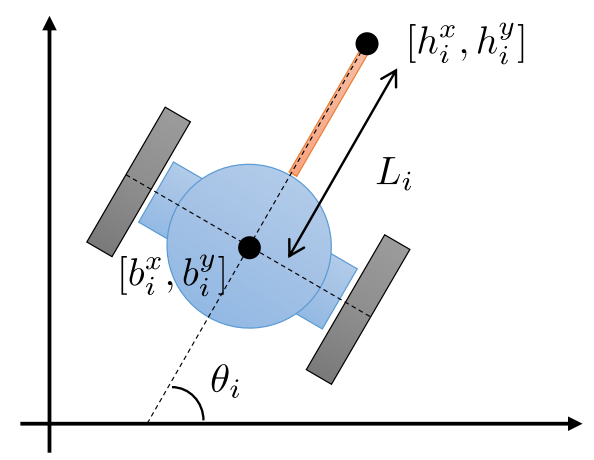

Figure 1. Unicycle model for the vehicle used for controlling the hand position

Letting $z_{i}=\left[b_{i}^{x}, b_{i}^{y}, \theta_{i}, s_{i}, \omega_{i}\right]^{T}$ and $\nu_{i}=\left[F_{i}, \tau_{i}\right]^{T}$, the dynamical equations can be easily written as:

$$
\dot{z}_{i}=f\left(z_{i}\right)+g_{i} \nu_{i}
$$

Considering the hand position of each vehicle as output, it is shown in Lawton et al. (2003) that system (10) has constant relative degree equal to two and can, therefore, be output feedback linearized about the hand position. Now, by using the next output feedback linearizing control:

$$
\nu_{i}=\left[\begin{array}{cc}
\frac{1}{m_{i}} \cos \left(\theta_{i}\right) & -\frac{L_{i}}{I_{i}} \sin \left(\theta_{i}\right) \\
\frac{1}{m_{i}} \sin \left(\theta_{i}\right) & \frac{L_{i}}{I_{i}} \cos \left(\theta_{i}\right)
\end{array}\right]^{-1}\left(u_{i}-\left[\begin{array}{c}
-s_{i} \omega_{i} \sin \left(\theta_{i}\right)-L_{i} \omega_{i}^{2} \cos \left(\theta_{i}\right) \\
s_{i} \omega_{i} \cos \left(\theta_{i}\right)-L_{i} \omega_{i}^{2} \sin \left(\theta_{i}\right)
\end{array}\right]\right),
$$

the input-output dynamics of each vehicle can be represented by the double integrator system:

$$
\ddot{h}_{i}=u_{i}
$$

It should be mentioned that the internal dynamics, related to the orientation of the vehicle itself, are unobservable and uncontrollable. However, it is also shown in Lawton 
et al. (2003) that this internal dynamics is stable, but not asymptotically stable. Therefore, we will assume that the orientation of each vehicle is locally measured.

\subsection{Agent description}

It is assumed that each vehicle is equipped with an agent that has sensing, control, computational and communication capacities. In what respect to the sensing ability, each agent $i$ is assumed to measure the following output:

$$
y_{i}(k)=C_{i} x(k)+v_{i}(k)
$$

where $y_{i} \in \mathbb{R}^{m_{i}}$ is output vector, $C_{i}$ is the output matrix and $v_{i}$ represents measurement noise. It is assumed that the noises are unknown, but they always belong to known bounded sets, that is, $v_{i}(k) \in \mathcal{V}_{i}$. These sets are modelled as:

$$
\mathcal{V}_{i}=\left\langle 0, R_{i}\right\rangle
$$

We distinguish two kinds of agents: a) those who are able to measure the absolute position-like variables of its local vehicle (by using GPS-like sensors or similar), and b) those who measure relative position-like variables (distances, with optical or acoustic sensors). Both kind of agents are assumed to get information concerning its local velocity-like variables. Matrix $C_{i}$, as supposed, is structurally different for each kind of agent.

Example 1. Consider $p=3$ vehicles moving in a one-dimensional space. Then,

$$
A_{v e h}^{c}=\left[\begin{array}{cc}
0 & 1 \\
a_{21}^{1} & a_{22}^{1}
\end{array}\right], B_{v e h}^{c}=\left[\begin{array}{l}
0 \\
1
\end{array}\right]
$$

and vector $x(k) \in \mathbb{R}^{6}$.

Let's assume that agent 1 is able to measure its own position, whereas agents 2 and 3 measure relative positions. Considering a graph topology given by $1 \leftrightarrow 2 \leftrightarrow 3$, matrices $C_{1}, C_{2}, C_{3}$ are given by:

$$
\begin{aligned}
C_{1} & =\left[\begin{array}{llllll}
1 & 0 & 0 & 0 & 0 & 0 \\
0 & 1 & 0 & 0 & 0 & 0
\end{array}\right], \\
C_{2} & =\left[\begin{array}{cccccc}
-1 & 0 & 1 & 0 & 0 & 0 \\
0 & 0 & 1 & 0 & -1 & 0 \\
0 & 0 & 0 & 1 & 0 & 0
\end{array}\right], \\
C_{3}= & {\left[\begin{array}{cccccc}
0 & 0 & -1 & 0 & 1 & 0 \\
0 & 0 & 0 & 0 & 0 & 1
\end{array}\right] . }
\end{aligned}
$$

The agents are endowed with communication devices that let them send/receive information to/from their neighbours. The communication topology is mathematically described by a graph $G$. It is assumed that two possible topologies are valid, chain or star. This assumption will be required later to solve the problem of distributed guaranteed estimation. For instance, for a four vehicle fleet, the two possible schemes 
are depicted in Figure $2^{2}$. The arrows represent the communication between the agents.

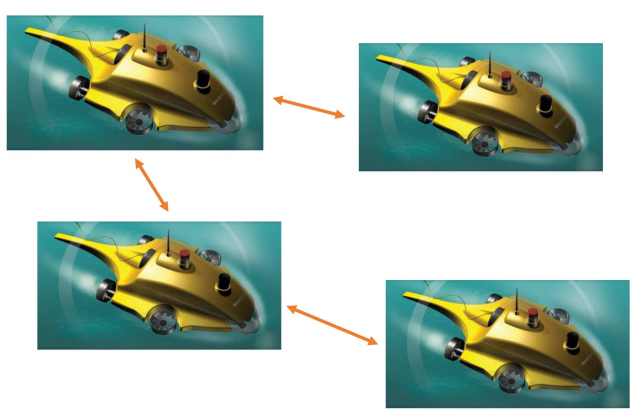

(a) Chain

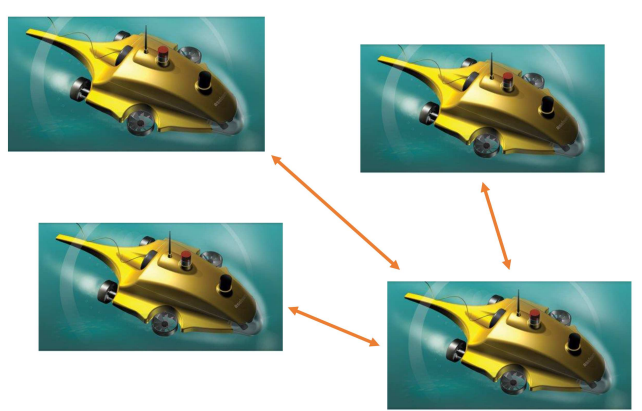

(b) Star

Figure 2. Scheme of the formation of the vehicles with the communication between agents

It is worth mentioning that those topologies only apply for the communication, not for the very formation of the vehicles. It will be shown in the examples that both topologies can be used to keep different formations.

By using the available information, that is, the measured outputs and the information received from the neighbourhood, the agents must compute estimations sets $\hat{\mathcal{X}}_{i}(k \mid k)$ within which the state must be contained. The construction of this estimation set is described in Section4.

Regarding the control of the vehicle, it is considered that each agent computes and applies the control signals to its local vehicle. The control signal applied by agent $i$ to the vehicle is chosen as:

$$
u_{i}(k)=K_{i} c_{i}(k \mid k),
$$

where the design of matrix $K_{i}$ will be tackled in Section 5 .

\subsection{Problem statement}

The goal of control formation problem is to keep a prescribed position for the vehicles. The global reference to be tracked $z_{r e f}(k) \in \mathbb{R}^{r \times p}$ is assumed to be known by each vehicle. Now, the problem can be formally stated.

Problem 1. Let's consider a fleet of $p$ vehicles described by the dynamical model (6). Consider an identical number of agents deployed together with the vehicles. Those agents get some outputs from the fleet given by (13). Let's assume that the disturbances and noises affecting the state and output are bounded by (7)-(14). The agents are able to exchange information according to a communication graph $G$, with chain or star topology. Given a set of position-like references for each vehicle $z_{r e f}(k)$, the objective of each agent is:

(1) Find an estimation set $\hat{\mathcal{X}}_{i}(k \mid k)$ such that $x(k) \in \hat{\mathcal{X}}_{i}(k \mid k)$ for all instant $k$.

(2) Design a local controller $K_{i}$ in such a way that the fleet is able to follow the reference $z_{r e f}(k)$.

\footnotetext{
${ }^{2}$ The image of the vehicle is obtained from the url: http://i-hls.com/wp-content/uploads/2013/08/ Autonomous-Underwater-Vehicle-AUV-Aselsan.jpg
} 
Compared to Orihuela et al. (2017), the estimation objective is here more complex, since in that paper the agents' goal was to estimate the state of its associated subsystem $x_{i}(k)$. The ignorance of the control actions applied by the rest of agents, that is, $u_{j}(k)$ for $j \neq i$, does not lead to a difficulty in the estimation problem formulated in Orihuela et al. (2017). However, in Problem 1, this fact appears as the main complication.

\section{Guaranteed distributed estimator}

This section presents the estimation algorithm that aims to satisfy objective (1) of Problem 1. Nevertheless, there are some common obstacles that arise in this sort of estimation and control problems that should be described firstly.

\subsection{Main obstacles and proposed solutions for the joint distributed control and estimation problem}

When facing the joint problem of distributed estimation and control, the control engineer finds two main drawbacks:

- Estimating the whole state with partial information: this difficulty is common in the literature of distributed estimation, no matter the sort of estimator used. The output measured by an agent does not contain enough information to estimate the whole state vector. Although the mathematical details vary between the different formulations, the solution typically consists in introducing the information received from neighbouring agents in the formulation of a centralized observer. For instance, distributed Kalman filter formulations modify the classical Kalman filter by adding the neighbouring information using consensus, as in Battistelli and Chisci (2016); Das and Moura (2015); del Nozal et al. (2017); Olfati-Saber (2007), or with diffusion strategies, see Cattivelli and Sayed (2010); G. Wang et al. (2017). Consensus algorithms have been also used to extend the Luenberger observer to a distributed formulation in del Nozal et al. (2019); Matei and Baras (2012); Mitra and Sundaram (2018), or for the distributed the Bayesian filter, see Forti et al. (2018).

The literature of distributed set-membership estimation is not that rich. However, some interesting solutions for this problem can be found. The information received from the neighbourhood can be incorporated in the estimated set by intersection, see García et al. (2017); Keiffer (2009), or by means of linear combinations of the sets, as in Combastel and Zolghadri (2018); Orihuela et al. (2017). Previous papers described the sets using zonotopes. Recently, it has been proposed the use of consensus techniques for distributed estimation with ellipsoidal sets, see Ma et al. (2017).

- Predicting with an unavailable control signal: this problem only appears when the joint distributed and estimation scheme is tackled at the same time. When a local observer has to make predictions, it must incorporate, at least, two terms: the dynamics of the system and the control actions applied to it. Although a perfect knowledge of the dynamics is assumed, how can an individual agent know the actual control actions if they might be applied by agents that might be geographically distributed?

The solutions adopted in Millán et al. (2013); Orihuela, Millán, Vivas, and Rubio (2016); Rubio et al. (2014); Zhang et al. (2017) present different formula- 
tions of a cascade structure, where the estimation errors must converge to zero to achieve the control objectives. In all these papers, the authors assume that the control actions applied to the system at any moment are the same that the local agent would apply if it has access to all control channels. This incurs in an error that dissapears when the estimated states of every agent converge to the system state.

This idea cannot be applied directly to set-membership paradigms, since the described assumption will lead to non-guaranteed estimations in the transient. The literature of distributed control and set-membership estimation is rather scarce, and it is limited to formulations of the plant divided into subsystems for which the estimation objectives are just local, see Combastel and Zolghadri (2018); Orihuela et al. (2018, 2017).

\subsection{Proposed guaranteed estimation algorithm}

This section presents the distributed estimation algorithm that will be executed by every agent deployed in the vehicles. In order to save the obstacle described before for the joint control and estimation problem, the proposed solution makes use of two key observations.

Recall that $\hat{\mathcal{X}}_{i}(k \mid k)$ stands for the estimation set (described by a zonotope) computed by agent $i$ at instant $k$ with all the information available at instant $k$. Let's denote by $\mathcal{Z}_{i}(k)$ to the zonotope sent by agent $i$ to all its neighbours. This zonotope differs, in general, with the estimation set computed by agent $i$. Then, if agent $j$ is going to use the zonotope received from agent $i$ at instant $k$ to obtain an estimation set $\hat{\mathcal{X}}_{j}(k \mid k)$ by intersection with $\mathcal{Z}_{i}(k)$, then agent $i$ knows that the estimation set of agent $j$ at instant $k$, at least, belong to $\mathcal{Z}_{i}(k)$. Hence, when agent $j$ computes and applies its local control action to the plant based on (15), agent $i$ will know a region into which the actual control action applied by $j$ is guaranteed to belong. This region will be used to construct the guaranteed prediction sets.

Nonetheless, observe that the previous mechanism is only valid for direct neighbours. To be applicable in general, an additional observation is required. When agent $i$ sends a zonotope $\mathcal{Z}_{i}(k)$ at instant $k$, the information carried in this set spreads through the network in direct paths (without loops) and will be received by every agent, at most, at instant $k+\bar{d}-1$. This is true due to the particular topologies considered. Since every agent $j \neq i$ will incorpore this information to construct their estimation set $\hat{\mathcal{X}}_{j}(k+\alpha \mid k+\alpha)$ by intersection, for some $\alpha \leq \bar{d}-1$, then agent $i$ will be able to compute a possible control region for the control action applied by agent $j$ at instant $k+\alpha$ by means of open-loop predictions.

Before introducing the mathematical details, an illustrative example is presented that will surely help to clarify these ideas.

Example 2. Consider $p=3$ vehicles with associated agents connected with a chain topology $1 \leftrightarrow 2 \leftrightarrow 3$. Consider that agent 3 applies a control action at instant $k+1$. Agent 1 requires to compute a region that contains for sure this control action $u_{3}(k+1)$.

Figure 3 depicts, by using illustrative colours and shapes, the way the information spreads through the network. The zonotope sent by agent 1 at instant $k$ (blue circle) affects the estimation set computed by agent 2 (brown triangle). Then, after an adequate evolution of this set (big brown triangle), it will affect the estimation set of agent 3 at instant $k+1$. Since the information of each set is used by means of 


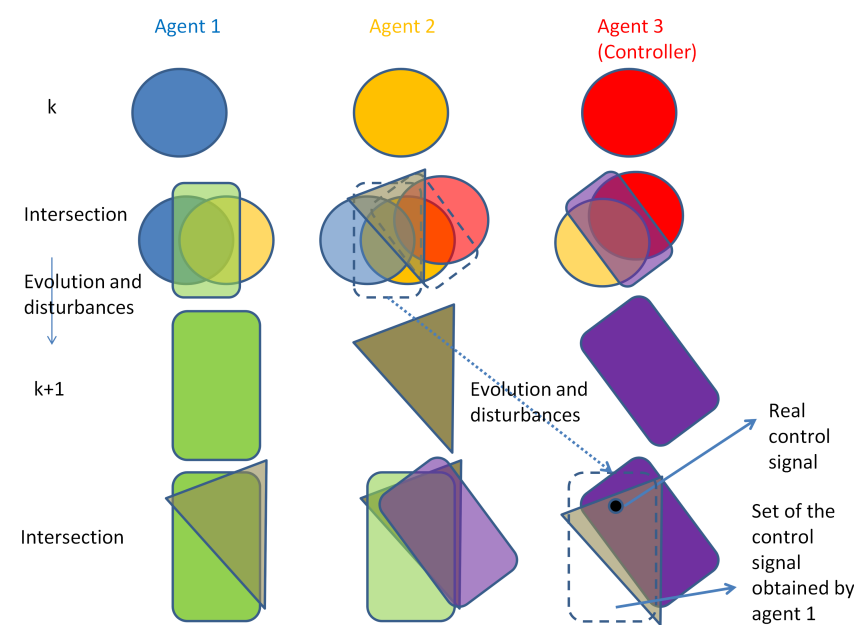

Figure 3. Illustrative example of the transmission of information through the network. Intersections and evolutions of the sets are not pretended to be exact

Table 1. Control and estimation loop

\begin{tabular}{ll}
\hline 0) Initial zonotope & $\hat{\mathcal{X}}_{i}(k \mid k-1)$ \\
\hline 1) Measurement & $y_{i}(k)$ \\
\hline 1.1) Strip & $S_{i}(k)$ \\
\hline 1.2) Intersection & $\mathcal{Z}_{i}(k) \triangleq \hat{\mathcal{X}}_{i}(k \mid k-1) \cap S_{i}(k)$ \\
\hline 1.3) Order reduction & $\operatorname{red}\left(\mathcal{Z}_{i}(k)\right)$ \\
\hline 2) Communication & \\
\hline 2.1) Send & $\operatorname{red}\left(\mathcal{Z}_{i}(k)\right)$, to all $j: i \in \mathcal{N}_{j}$ \\
\hline 2.2) Receive & $\operatorname{red}\left(\mathcal{Z}_{j}(k)\right), \forall j \in \mathcal{N}_{i}$ \\
\hline 2.3) Compute & $\mathcal{I}_{i j}(k) \triangleq \operatorname{red}\left(\mathcal{Z}_{i}(k)\right) \cap \operatorname{red}\left(\mathcal{Z}_{j}(k)\right)$ \\
\hline 3) Estimation & $\hat{\mathcal{X}}_{i}(k \mid k)=\bigcap_{j} \mathcal{I}_{i j}, \forall j \in \mathcal{N}_{i}$ \\
\hline 4) Control & $u_{i}(k)=K_{i} c_{i}(k \mid k)$ \\
\hline 5) Prediction & $\hat{\mathcal{X}}_{i}(k+1 \mid k)=A \hat{\mathcal{X}}_{i}(k \mid k) \oplus B \hat{\mathcal{U}}_{i}(k) \oplus \mathcal{W}$ \\
\hline
\end{tabular}

intersections, agent 1 will be able to evolve its estimation set at instant $k$ (green rectangle) in open loop to obtain a predicted set (big green rectangle) that contains the actual control action $u_{3}(k+1)$.

The complete control and estimation algorithm performed by every agent has five main steps. Next, we provide a description of this algorithm, which has been summarized in Table 1.

1. Measurement: using the measured outputs, $y_{i}(k)$ in (13), the agents compute a strip of consistent states with each component of this vector, $S_{i}^{l}(k)$, for $l=1 \ldots, m_{i}$. These strips are defined as in Section 2. In particular, considering for example a unidimensional output, the strip will be given by $S_{i}(k)=\left\{x(k):\left|C_{i} x(k)-y_{i}(k)\right|<R_{i}\right\}$, where $R_{i}$ is related with the output noise, see (14). Note that the width of this strip directly grows with the energy of the measurement noise. Then, the prediction zonotope available from the last time step $\hat{\mathcal{X}}_{i}(k \mid k-1)$ is intersected with every $S_{i}^{l}(k)$, obtaining $\mathcal{Z}_{i}(k)$ as a final result. The intersection between a strip and a zonotope can be written 
as a zonotope, as was explained in Section 2. Finally, an order reduction operation is executed to obtain zonotope $\operatorname{red}\left(\mathcal{Z}_{i}(k)\right)$.

2. Communication: at this step of the algorithm the agents send the reduced-order zonotopes obtained before to all its neighbours. After that communication, each pair of connected agents computes the intersection zonotope $\mathcal{I}_{i j}(k) \triangleq \operatorname{red}\left(\mathcal{Z}_{i}(k)\right) \cap \operatorname{red}\left(\mathcal{Z}_{j}(k)\right)$, which allows them to reduce the estimation uncertainty. Observe that $\mathcal{I}_{i j}(k)=\mathcal{I}_{j i}(k)$, as they are related to the same edge in an undirected graph. As explained in Section 2, the intersection between zonotopes can be written as a zonotope.

3. Estimation: by including the information of every edge (in other words, of every neighbour), the agents obtain the best estimation set with the information available at instant $k$, that is, $\hat{\mathcal{X}}_{i}(k \mid k)$.

4. Control: the control action applied by agent $i$ to its vehicle is obtained using the center of the estimation set according to (15).

5. Prediction: finally, the prediction sets $\hat{\mathcal{X}}_{i}(k+1 \mid k)$ are computed as:

$$
\hat{\mathcal{X}}_{i}(k+1 \mid k)=A \hat{\mathcal{X}}_{i}(k \mid k) \oplus B \hat{\mathcal{U}}_{i}(k) \oplus \mathcal{W} .
$$

Three terms appear in the previous equation, one for each term in (8): 1) the openloop dynamics of the system, with the term $\left.A \hat{\mathcal{X}}_{i}(k \mid k) ; 2\right)$ the set of possible control signals applied to the plant, $\left.B \hat{\mathcal{U}}_{i}(k) ; 3\right)$ and the set of all possible disturbances, given by $\mathcal{W} \triangleq\left[\mathcal{W}_{1}^{T} \mathcal{W}_{2}^{T} \cdots \mathcal{W}_{p}^{T}\right]^{T}$. The set $\hat{\mathcal{U}}_{i}(k)$ should contain every control action applied to the plant at instant $k$. Next, we present a method to compute this set.

Note that $B u(k)$ can be written as $\sum_{m=1}^{p} B_{i} u_{i}(k)$, where $B_{i}$ is the submatrix of $B$ for the control channels of agent $i$. With a little abuse of notation, let's denote $\hat{\mathcal{U}}_{i}(k)=\left[\hat{\mathcal{U}}_{i}^{m}(k)\right]$, where $\hat{\mathcal{U}}_{i}^{m}(k)$ is a set computed by agent $i$ that must contain the vector $u_{m}(k)$, and it is given by:

$$
\hat{\mathcal{U}}_{i}^{m}(k) \triangleq\left\{\begin{aligned}
(a): \quad & \text { if } m=i \\
& K_{i} c_{i}(k \mid k) \\
(b): & \text { if } m \neq i, m \in \mathcal{N}_{i} \\
& K_{m} \mathcal{I}_{i m}(k) \\
(c): \quad \text { otherwise } & K_{m} \hat{\mathcal{I}}_{i m}\left(k \mid k-d_{i m}+1\right),
\end{aligned}\right.
$$

being $\hat{\mathcal{I}}_{i m}\left(k \mid k-d_{i m}+1\right)$ the prediction of the intersection zonotope computed as:

$$
\begin{aligned}
& \hat{\mathcal{I}}_{i m}\left(k \mid k-d_{i m}+1\right)=A^{d_{i m}-1} \mathcal{I}_{i j}\left(k-d_{i m}+1\right) \oplus \bigoplus_{n=1}^{d_{i m}-1} A^{n-1} B \hat{\mathcal{U}}_{i}(k-n) \\
& \oplus\left[\sum_{n=1}^{d_{i m}-1} A^{n-1}\right] \mathcal{W}
\end{aligned}
$$

where $j \in \mathcal{N}_{i}$ is a neighbour of agent $i$ such that there exists a path from $m$ to $j$. Observer that the zonotope $\hat{\mathcal{I}}_{i m}\left(k \mid k-d_{i m}+1\right)$ represents the open-loop evolution of the zonotope $\hat{\mathcal{I}}_{i j}$ for $d_{i m}-1$ time instants, which is the number of instants required for the zonotope $\hat{\mathcal{I}}_{i j}$ to reach agent $m$.

Please note that for $m \in \mathcal{N}_{i}$ expression (17)-(b) matches (17)-(c), for $d_{i m}=1$. Nonetheless, three cases has been defined to distinguish between three important sit- 
uations: $(a)$ agent $i$ applies its local control signal $u_{i} ;(b)$ agent $i$ is a neighbour of the agent with access to the control channel; and $(c)$ agent $i$ is not neighbour of the agent applying $u_{m}$.

Next theorem states that, with the proposed algorithm, objetive (1) of Problem 1 is met, that is, every agent is able to compute estimation sets containing the actual state vector of the whole fleet of vehicles.

Theorem 1. Assume that $x\left(k_{0}\right) \in \hat{\mathcal{X}}_{i}\left(k_{0} \mid k_{0}-1\right)$ holds for all agents $i=1,2, \ldots p$ at some arbitrary initial instant $k_{0}$. Also assume that $x\left(k_{0}-t\right) \in \hat{\mathcal{X}}_{i}\left(k_{0}-t \mid k_{0}-t\right)$ and $u\left(k_{0}-t\right) \in \hat{\mathcal{U}}_{i}\left(k_{0}-t\right)$ holds for all agents $i=1,2, \ldots, p$ and for $t=1,2, \ldots, d_{\text {max }}$. Then, if each agent implements the distributed algorithm described in Table 1, it is satisfied that $x(k) \in \hat{\mathcal{X}}_{i}(k \mid k)$ and $x(k+1) \in \hat{\mathcal{X}}_{i}(k+1 \mid k), \forall k, i$.

Proof. The theorem will be proved by induction. Assume, hence, that $x(k) \in \hat{\mathcal{X}}_{i}(k \mid k-$ $1), \forall i$.

The first part of the proof focuses on the estimation set $\hat{\mathcal{X}}_{i}(k \mid k)$. By construction, the strip $S_{i}(k)$ is the set consistent with the measurement, so $x(k) \in S_{i}(k)$. Since zonotope $\mathcal{Z}_{i}(k)$ is the representation of the intersection of $S_{i}(k)$ and $\hat{\mathcal{X}}_{i}(k \mid k-1)$, then $x(k) \in \mathcal{Z}_{i}(k)$. The order-reduction operation verifies that $\mathcal{Z}_{i}(k) \subseteq \operatorname{red}\left(\mathcal{Z}_{i}(k)\right)$, so $x(k) \in \operatorname{red}\left(\mathcal{Z}_{i}(k)\right), \forall i$.

It is trivially true that $x(k) \in \mathcal{I}_{i j}$ for the same reasons exposed above. As zonotope $\hat{\mathcal{X}}_{i}(k \mid k)$ is computed as the intersection of several $\mathcal{I}_{i j}$ (as many as neighbours), then it holds $x(k) \in \hat{\mathcal{X}}_{i}(k \mid k)$.

Now, let's move to the second part of the theorem that focuses on the prediction set. By comparing both equations (8) and (16), it is easy to check that $A x(k) \in A \hat{\mathcal{X}}_{i}(k \mid k)$ and $w(k) \in \mathcal{W}$. Next, it will be proven that $u_{m}(k) \in \hat{\mathcal{U}}_{i}^{m}(k), \forall m$.

For $m=i$ it is trivially true, since $\hat{\mathcal{U}}_{i}^{i}(k)=u_{i}(k)$. For $m \in \mathcal{N}_{i}$, note that $u_{m}(k)=$ $K_{m} c_{m}(k \mid k)$, and $c_{m}(k \mid k)$ is the centre of the estimation zonotope $\hat{\mathcal{X}}_{m}(k \mid k)$. Zonotope $\hat{\mathcal{X}}_{m}(k \mid k)$ is obtained by the intersection of all the $\mathcal{I}_{m j}(k), \forall j \in \mathcal{N}_{m}$. In particular, $\mathcal{I}_{i m}(k)$ is one of those sets, so $\hat{\mathcal{X}}_{m}(k \mid k) \subseteq \mathcal{I}_{m i}(k)=\mathcal{I}_{i m}(k)$. Then, $c_{m}(k \mid k) \in \mathcal{I}_{\text {im }}(k)$ and, therefore $u_{m}(k) \in \hat{\mathcal{U}}_{i}^{m}(k)$.

Finally, consider the last case, whose proof is based on how the information travels though the communication network. The second assumption says that $x(k-t) \in$ $\hat{\mathcal{X}}_{i}(k-t \mid k-t)$ for all the agents, and for $t=1,2, \ldots d_{\max }-1$, and by definition of $\hat{\mathcal{X}}_{i}(k-t \mid k-t)$ that can be expressed as $x(k-t) \in \mathcal{I}_{i j}(k-t)$. It also says that $u(k-t) \in \hat{\mathcal{U}}_{i}(k-t)$ for all the agents, and for $t=1,2, \ldots d_{\text {max }}$. Summarizing, if it is known a set where the state was in a known instant, $\mathcal{I}_{i j}\left(k-d_{i m}+1\right)$, the control signals applied and the set of disturbances, then the state at instant $k$ belongs to $\hat{\mathcal{I}}_{i m}\left(k \mid k-d_{i m}+1\right)$.

The set $\mathcal{I}_{i j}\left(k-d_{i m}+1\right)$ travel through the network and affects to $\hat{\mathcal{X}}_{m}(k \mid k)$ in an undirected way due to the interactions among the agents of the network (intersection of zonotopes). Its evolution due to matrix $A$ gives a bigger set than $\hat{\mathcal{X}}_{m}(k \mid k)$ since it is not corrected with measurements or communication with other agents, and then the set of action signals and the set of disturbances are added in order to compute a set that is affected in the same way than the state. So the centre $c_{m}(k \mid k) \in \hat{\mathcal{I}}_{i m}\left(k \mid k-d_{i m}+1\right)$ and then $u_{m}(k) \in \hat{\mathcal{U}}_{i}^{m}(k)$.

Then, by properties of the Minkowski sum of sets, it is proved that $x(k+1) \in$ $\hat{\mathcal{X}}_{i}(k+1 \mid k)$ for all $i$. Since $x\left(k_{0}\right) \in \hat{\mathcal{X}}_{i}\left(k_{0} \mid k_{0}-1\right)$ was required by assumptions, the 
theorem is proved by induction.

The hypothesis of Theorem 1 are standard for induction-based proofs. Moreover, they can be easily fulfilled since the initial sets, namely $\hat{\mathcal{X}}_{i}\left(k_{0} \mid k_{0}-1\right), \hat{\mathcal{X}}_{i}\left(k_{0}-t \mid k_{0}-\right.$ $t), \hat{\mathcal{U}}_{i}\left(k_{0}-t \mid k_{0}-t\right)$ for $t=1,2, \ldots, d_{\text {max }}$, can be trivially enlarged so that they contain for sure the required state vector. Another initialization scheme consists in a preliminar execution of the algorithm for $d_{\max }$ time instants with $u_{i}(k) \equiv 0$ (open-loop dynamics). Then, it will be easily satisfied that $u\left(k_{0}-t\right) \in \hat{\mathcal{U}}_{i}\left(k_{0}-t\right)$ for $t=1,2, \ldots, d_{\text {max }}$. After this initial steps, the algorithm can run in its proposed manner.

Theorem 1 proves that every agent is able to find sets that contain for sure the actual state for the plant. However, it did not directly mention that these sets are found in an optimal way. In fact, the reader might think that the sets could grow until infinity, in such a way that the theorem would be fulfilled but the estimation would be inadequate.

Nevertheless, two main facts prevent the uncontrolled growing of the estimation sets. On the one hand, the mathematical operations involving zonotopes, such as intersection with strip, intersection between zonotopes and order reduction, are computed using published methods that ensure the optimality of those operations (see Section 2 and the references therein). On the other hand, under standard observability assumption, all the outputs collected by the whole fleet contain enough information to estimate the complete state. Since the algorithm is based in the inclusion of this information by means of intersections, the estimation sets are always kept under some bounds.

Finally, it is worth mentioning that the prediction step (Step 5 in Table 1) must be deeply analyse in the future, since its performance can be improved if it takes into consideration the particular topology. It is left for future research.

\section{Control formation}

In the following, we briefly describe how to track position references in a space state representation, inspired on Orihuela et al. (2016). Given (8), a new system's output is set as $z(k)=C_{z} x(k)$, where $C_{z}$ is a matrix that selects the position ( $X$ and $Y$ ) of each vehicle. On the other hand, the position references are given by the vector $z_{r e f}(k)$, which in general, might take different values for different instants. The equilibrium point $\left(x_{e q}(k), u_{e q}(k)\right)$ of system (8) (without disturbances) associated with the reference $z_{r e f}(k)$ at instant $k$ must satisfy

$$
\begin{gathered}
x_{e q}(k)=A x_{e q}(k)+B u_{e q}(k), \\
z_{r e f}(k)=C_{z} x_{e q}(k),
\end{gathered}
$$

which can be expressed as:

$$
\left[\begin{array}{c}
0 \\
z_{r e f}(k)
\end{array}\right]=\left[\begin{array}{cc}
A-I & B \\
C_{z} & 0
\end{array}\right]\left[\begin{array}{l}
x_{e q}(k) \\
u_{e q}(k)
\end{array}\right]
$$

According to previous equation, the equilibrium point associated with $z_{r e f}(k)$ can 
be obtained as

$$
\left[\begin{array}{l}
x_{e q}(k) \\
u_{e q}(k)
\end{array}\right]=\left[\begin{array}{cc}
A-I & B \\
C_{z} & 0
\end{array}\right]^{-1}\left[\begin{array}{c}
0 \\
z_{r e f}(k)
\end{array}\right]
$$

Then, in order to track the reference $z_{r e f}(k)$, the following linear system must be stabilised

$$
x_{r}(k+1)=A x_{r}(k)+B u_{r}(k),
$$

where $x_{r}(k)=x(k)-x_{e q}(k)$ and $u_{r}(k)=u(k)-u_{e q}(k)$. Previous system is completely equivalent to the one given (8).

Concerning the design of the local controllers $K_{i}$, note that each vehicle has its own estimation about the positions and velocities of the whole fleet. Furthermore, observe that matrices $A, B$ in (22) decouple the fleet control formation problem into $p$ local control problems, assuming that each vehicle knows its own reference. Let's denote $\left(x_{e q}^{i}(k), u_{e q}^{i}(k)\right)$ as the equilibrium point for vehicle $i$ related to the global reference $z_{r e f}(k)$. Now, let's define the following local cost function:

$J_{i}=\sum_{k=0}^{\infty}\left(c_{i}(k \mid k)-x_{e q}^{i}(k)\right)^{T} \Phi_{i}\left(c_{i}(k \mid k)-x_{e q}^{i}(k)\right)+\left(u_{i}(k)-u_{e q}^{i}(k)\right)^{T} \Theta_{i}\left(u_{i}(k)-u_{e q}^{i}(k)\right)$,

being $\Phi_{i}, \Theta_{i}$ two positive-definite matrices. The local controller is then found by finding the gain $K_{i}$ that minimizes the previous cost function, following a LQR-based paradigm with the controllable pair $\left(A_{v e h}, B_{v e h}\right)$.

\section{Simulation examples}

The performance of the algorithm is illustrated with several simulations with a fleet of 4 vehicles. The dynamics of each of them in (5) are particularized as a double integrator and in a 2D scenario. Table 2 presents the set of common parameters that have been chosen for the simulations. The rest of parameters, such as the weighting matrices for the LQR cost function or the noise zonotopes, will be given in the different examples, since they will take different values for each experiment.

Table 2. Parameters for the simulations

\begin{tabular}{ll}
\hline Configuration variables & 2 \\
\hline Vehicle dynamics & $a_{21}^{r}=0, a_{22}^{r}=0, r=1,2$ \\
\hline Sampling time & $T_{s}=0.1 s$ \\
\hline Maximum order & 200 \\
\hline
\end{tabular}

\subsection{Example 1}

We consider a fleet of four vehicles, being the vehicle 1 the one that is able to measure its absolute position, and the rest measure relative positions. We will compare both topologies considered in the paper. The communication topology, then, will be different in each case: 
Chain $\operatorname{veh}_{1} \leftrightarrow \operatorname{veh}_{2} \leftrightarrow \operatorname{veh}_{3} \leftrightarrow \operatorname{veh}_{4}$

Star $\operatorname{veh}_{1} \leftrightarrow \operatorname{veh}_{2}, \operatorname{veh}_{1} \leftrightarrow \operatorname{veh}_{3}, \operatorname{veh}_{1} \leftrightarrow \operatorname{veh}_{4}$

This example will show that the fleet is able to keep a given formation (which indeed is different to a linear or star formation) when the proposed algorithm is used. In addition, all the agents will be able to compute adequate estimation sets for the whole fleet. We assume, as in Lafferriere et al. (2005), that the disturbances affecting the model are equal to zero. Measurement noises are however taken into consideration. It is also assumed that the center of the initial zonotopes is located exactly where the initial state of the vehicles is.

Without loss of generality, the reference trajectory is divided into four waypoints (see Section 5). Figures 4a-4b depict, for both situations, the actual trajectories of the vehicles on the plane $x y$ using solid lines. The initial position is marked with a circle, and the final position with a triangle. The references are represented in dashed lines. It can be seen that the proposed control formation obtains very nice results. No remarkable differences can be found between both scenarios in what respect to control formation.

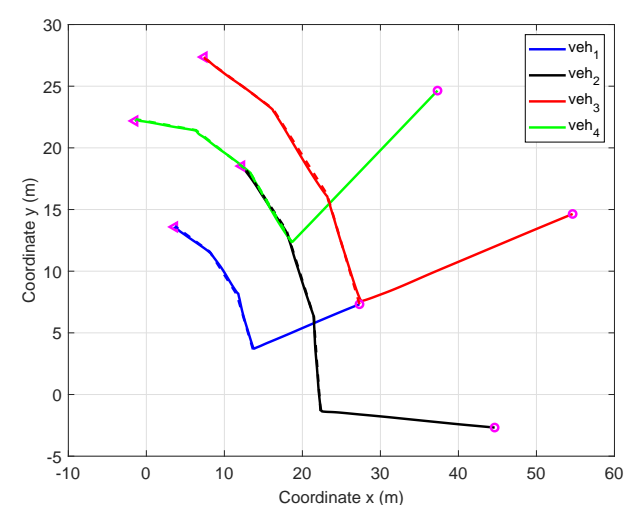

(a) Chain

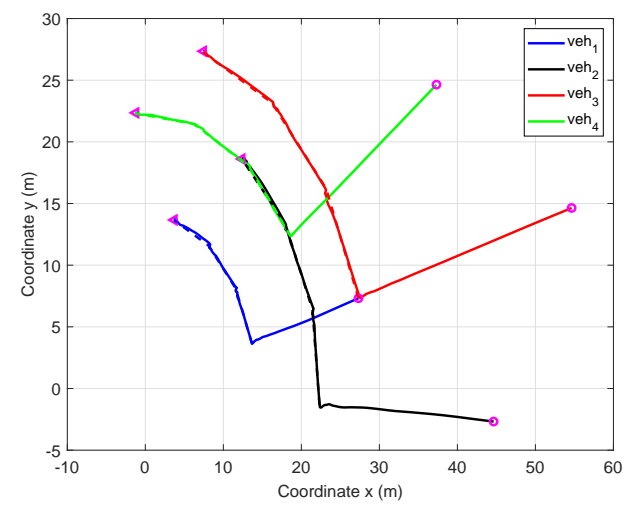

(b) Star

Figure 4. Trajectory of the four vehicles considering both communication topologies

On the other hand, the estimation performance can be checked in Figures 5a-5b. The figures depict the horizontal position of the vehicle 1 (black solid line), its reference (blue solid line), and the estimation that every agent computes. Note that the zonotopes represent an interval when considering a unique variable (in this case, the horizontal position), and so, minimum and maximum values for the state are depicted by each agent (dashed lines of different colours).

It is easy to check that the state is always contained within the sets that all agents compute, this showing that the first requirement of a guaranteed estimator is fulfilled. Figure 5 also shows that the settling time of the tracking is around 40 sampling instants (4 seconds) with the chosen controller for both cases.

In addition, the example illustrates the effect of the distance in the estimation when the chain topology is considered, in the sense that the farther an agent is to the agent measuring the particular state (in this case, agent 1), the wider its bounds are. The center of the zonotopes (the best estimation) does not suffer from this effect. On the contrary, for the star topology, all the agents can be considered symmetric and, therefore, they obtain a similar performance. 


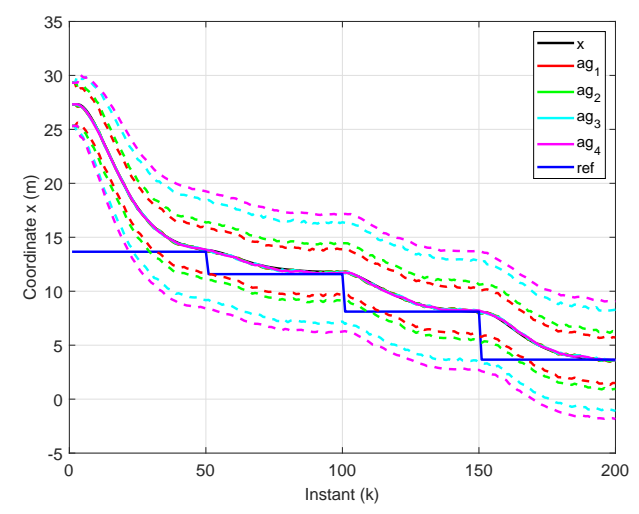

(a) Chain

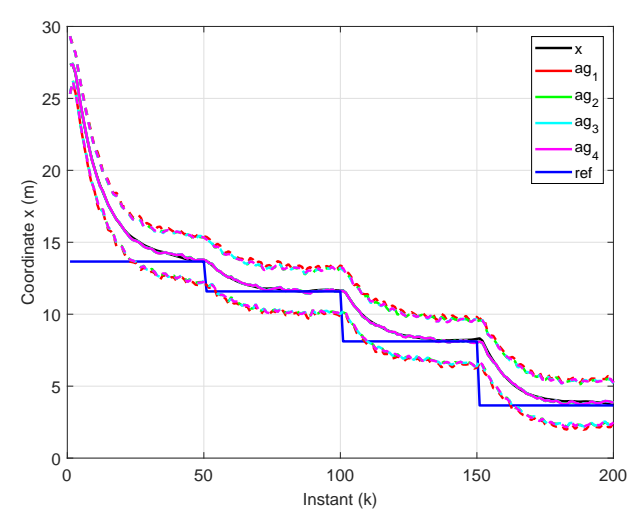

(b) Star

Figure 5. Vehicle 1, actual horizontal position, reference and estimation of the position $x$ by all agents

\subsection{Example 2}

This simulation example shows that the proposed distributed estimation and control method can be used for the nonlinear model of the unicycle, as long as the control of the hand is pursued.

We will assume, for the sake of simplicity, that the masses, moments of inertia and hand positions are the same for every vehicle, this is, $m_{i}=1, I_{i}=1, L_{i}=$ $1, \forall i$. Furthermore, the bounds of noises and disturbances are also the same, $\mathcal{W}_{i}=$ $\langle 0,0.05\rangle, \mathcal{V}_{i}=\langle 0,0.1\rangle$. A chain topology is considered for the communication between the vehicles.

In order to compute the output feedback linearizing control (11), it is assumed that each vehicle is equipped with an additional sensor that measures its local orientation $\theta_{i}$. The rest of variables, namely, $s_{i}, \omega_{i}$ can be obtained with the information of the estimation sets. The local cost functions in (23) are defined with $\Phi_{i}=0.1 I, \Theta_{i}=I, \forall i$,

Figures $6 \mathrm{a}-6 \mathrm{~b}$ depicts, respectively, the position of the hand and the position of the vehicle. Starting for different initial positions, the fleet is trying to join their hands at the origin. These figures effectively show how the proposed method is still valid for the control of the hand position of the fleet. The effect of the internal dynamics is clear in Figure 6b.

Figure $6 \mathrm{~b}$ also shows the bounds for the coordinate $y$ obtained with the setmembership estimator. Similarly that in the previous example, wider bounds are obtained for the vehicles located far from the leader. Although it is possible to obtain bounds for the coordinate $x$ as well, we have not included them in the figure to make it clearer for the reader. Note that the bounds could be used for collision avoidance. In particular, vehicle 2 and 3 might have a collision after some time when their bounds intersect each other.

\subsection{Example 3}

In this new example, we study the influence that the disturbances have in the estimation performance. The analysis is made considering again a chain communication topology, which increases the uncertainty. Since we are interested in the estimation bounds, we will assume that initial zonotopes are centered in the actual initial state 


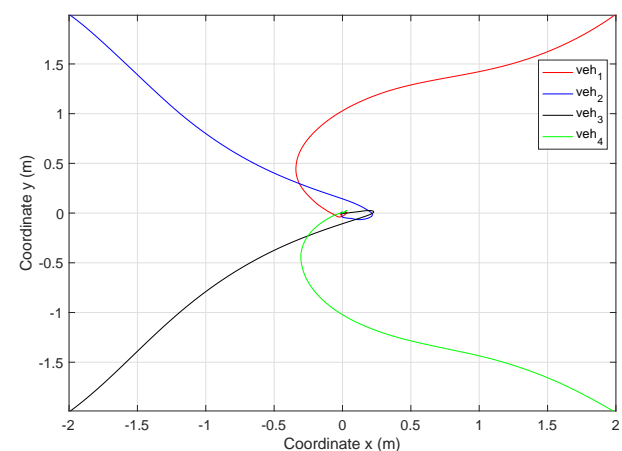

(a) Hand position

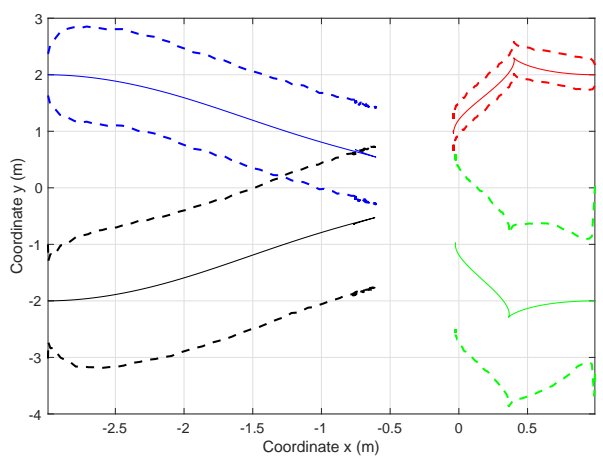

(b) Vehicle position

Figure 6. Position of the hand and vehicle considering the unicycle model

of the vehicles.

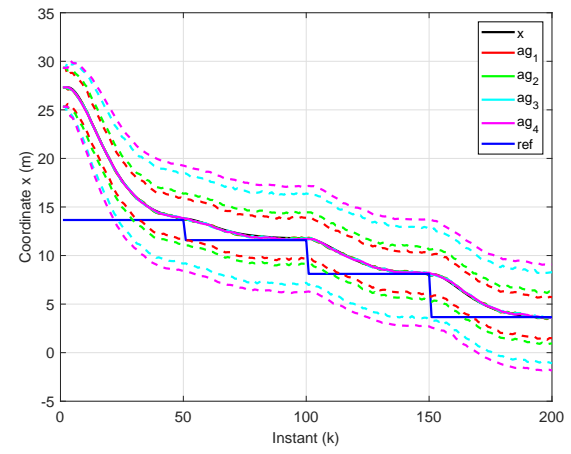

(a) Without disturbances

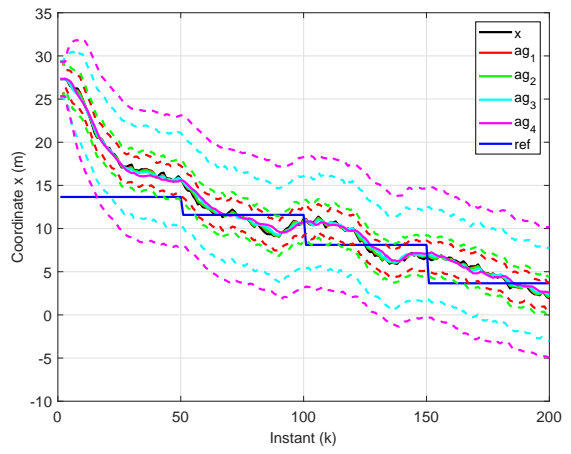

(b) With disturbances

Figure 7. Comparative in the chain communication scenario between the simulation without disturbances and with them

Two scenarios are compared in Figure 7 . In both cases, the observers consider that the disturbances might affect the vehicles, so zonotopes $\mathcal{W}_{i}=\langle 0,0.2 I\rangle, \forall i$, in $(7)$ are included in the prediction step. Whereas Figure 7a corresponds to the case in which no actual disturbances are applied, Figure $7 \mathrm{~b}$ shows the case in which the disturbances are actually affecting the dynamics.

It can be seen that the proposed control and estimation algorithm is able to track the prescribed reference in spite the fact that the estimation is degraded. Although the control signal applied by the rest of the fleet is ignored and the disturbances are affecting the vehicles, the guaranteed estimation is achieved.

The reader might think, from the previous simulations, that the reference is not tracked in the presence of disturbances. Then, we have made a longer simulation, depicted in Figure 8, that shows that the actual position of the vehicles evolve around the equilibrium point, within a confined region. The size of this region is related, as expected, to the energy of the disturbances. 


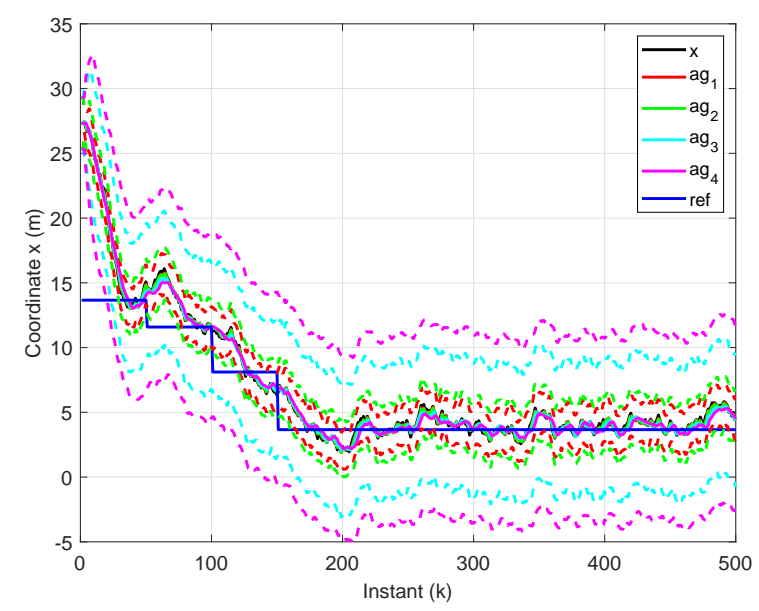

Figure 8. Vehicle 1, actual position, reference and bounds of the estimation of the position $x$ by all agents

\subsection{Example 4}

This example analyse the relation between the control matrix $K_{i}$ designed and the estimation performance. We will consider two identical settings (same initial condition, no noises, no disturbances) to perform a fair comparison. Although no noises and disturbances actually affect the system, the agents will take into account the corresponding zonotopes. In particular, $\mathcal{W}_{i}=\langle 0,0.1 I\rangle, \mathcal{V}_{i}=\langle 0,0.1 I\rangle, \forall i$. In addition, we will consider in this example that the initial position of the vehicles is unknown, so all the observers' initial condition is set to zero and the initial zonotope $\hat{\mathcal{X}}_{i}(0 \mid-1)=\langle 0,2.5 I\rangle$ is built big enought to encompass the initial state of the vehicles. This fact will let us compare the speed fo convergence of the estimation with respect to the control.

Two families of LQR controller have been synthesised, with different aggressiveness. In particular, the local cost functions in (23) are defined with $\Phi_{i}=10 I, \Theta_{i}=I, \forall i$, in the first scenario, and $\Phi_{i}=1 I, \Theta_{i}=I, \forall i$, in the second one. A chain communication topology has been considered here.

The results are illustrated in Figure 9 using black color lines for the first scenario and red color lines for the second one. The figures show the actual state in solid lines, the center of the estimation with circles, and the bounds with dashed lines. They all show the estimation of the vertical position of the first vehicle.

Several conclusions can be drawn from this set of figures:

- As in the previous results, the closer an agent is respect to the agent measuring the variable under consideration, the narrower the estimation is.

- The very first instants, in which the dynamics are frozen, represent the initialization procedure described after Theorem 1.

- The speed of convergence of the estimators is much faster than that of the controllers, this implementing the standard cascade structure shown by other combined algorithms, such as LQG.

- The values of the weighting matrices in the cost functions have a double impact: first of all, they let us tune the speed of convergence to the desired reference. However, the more aggressive the controllers are, the wider the estimation bounds become. This is due to the calculations made in the prediction step (16), that uses the controller matrices of the neighbours to compute the predicted zonotope. 
More aggressive controller means bigger values for the control matrices $K_{i}$ and, then, bigger prediction zonotopes following equation (17). As expected, this effect does not appear in the first agent, because this is the one measuring the vertical position.

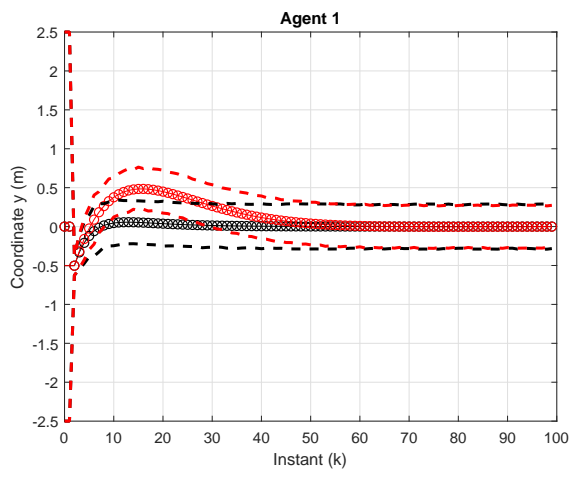

(a) Agent 1

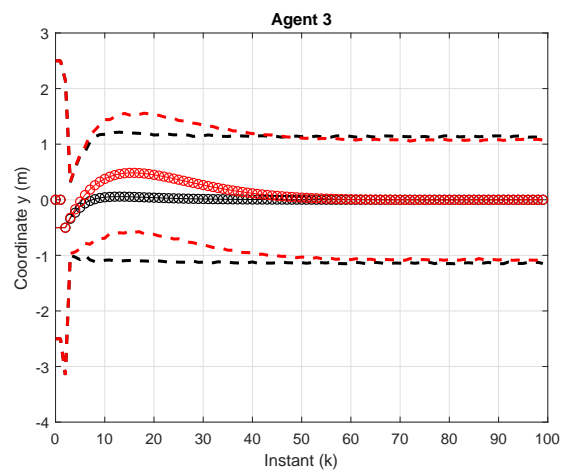

(c) Agent 3

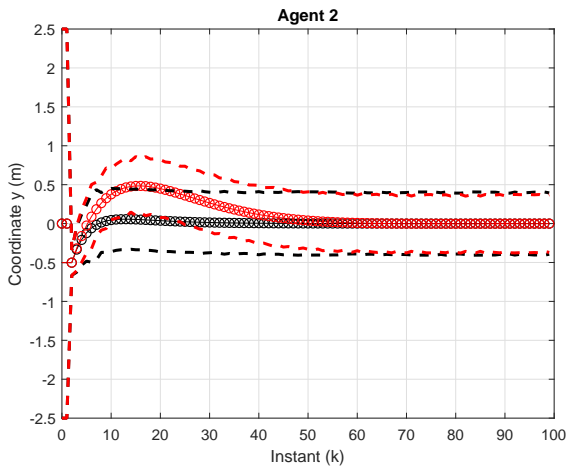

(b) Agent 2

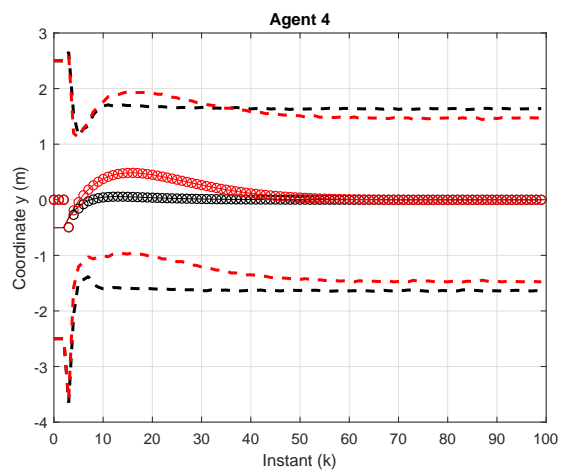

(d) Agent 4

Figure 9. Comparative of the gain $K_{i}$ based on a more aggressive control and on a less aggressive control, estimation of state 1 by all agents.

\section{Conclusions}

This paper proposes a set-membership distributed estimation and control algorithm. This approach assumes not only uncertainties in the model and in the measurement, but also lack of knowledge about the signal control and about the complete plant by the agents.

It has been tested on two different topologies, chain and star communication. The star communication obtains better results, since the maximum distance in the network is smaller than in the chain case. However, the relative distance between the central vehicle and each of the neighbour must belong within radio range. The extension of the proposed algorithm to other topologies, specially those containing a spanning tree (so that a directed path from every agent to any node with control capacities exists), is a natural extension of this work.

The impact of the controller design method in the estimation performance, and viceversa, must also be analyzed in the future. How is the control performance affected 
by the uncertainty in the estimation? How could the control matrix $K_{i}$ be designed with the combined objective of control and estimation performances? The answers to these questions will be subject of future research. Furthermore, considering unreliable links (with delays and dropouts) is another key problem in this field that will tackled in the future.

\section{Acknowledgments}

L. Orihuela and P. Millán have been supported by grant TEC2016-80242-P founded by AEI/FEDER through the Laboratorio de Simulación hardware-in-the-loop de Sistemas Ciberfísicos (LaSSiC). R.A. García has received funding from the European Research Council (ERC) under the European Unions Horizon 2020 research and innovation programme (grant agreement No 789051).

\section{References}

Achtelik, M. W., Lynen, S., Weiss, S., Chli, M., \& Siegwart, R. (2014). Motion-and uncertaintyaware path planning for micro aerial vehicles. Journal of Field Robotics, 31(4), 676-698.

Alamo, T., Bravo, J. M., \& Camacho, E. F. (2005). Guaranteed state estimation by zonotopes. Automatica, 41(6), 1035-1043.

Battistelli, G., \& Chisci, L. (2016). Stability of consensus extended Kalman filter for distributed state estimation. Automatica, 68, 169-178.

Bencini, L., Collodi, G., Di Palma, D., Manes, A., \& Manes, G. (2009, June). Advanced distributed monitoring system for agriculture based on wireless sensor network technology. In 7th world congress on computers in agriculture and natural resources (p. 330-335).

Cai, X., \& De Queiroz, M. (2015). Adaptive rigidity-based formation control for multirobotic vehicles with dynamics. Control Systems Technology, IEEE Transactions on, 23(1), 389396.

Cattivelli, F., \& Sayed, A. (2010). Diffusion strategies for distributed Kalman filtering and smoothing. IEEE Transactions on automatic control, 55(9), 2069-2084.

Chabane, S. B., Maniu, C. S., Alamo, T., Camacho, E. F., \& Dumur, D. (2014). A new approach for guaranteed ellipsoidal state estimation. In Ifac 2014 (pp. CD-ROM).

Combastel, C. (2015). Zonotopes and kalman observers: Gain optimality under distinct uncertainty paradigms and robust convergence. Automatica, 55, 265-273.

Combastel, C. (2016). An extended zonotopic and gaussian kalman filter (ezgkf) merging set-membership and stochastic paradigms: Toward non-linear filtering and fault detection. Annual Reviews in Control, 42, $232-243$.

Combastel, C., \& Zolghadri, A. (2018, August). FDI in cyber physical systems: A distributed zonotopic and Gaussian Kalman filter with bit-level reduction. In 10th ifac symposium on fault detection, supervision and safety for technical processes. Warsaw, Poland.

Das, S., \& Moura, J. (2015). Distributed Kalman filtering with dynamic observations consensus. IEEE Transactions on Signal Processing, 63(17), 4458-4473.

del Nozal, A., Millán, P., Orihuela, L., Seuret, A., \& Zaccarian, L. (2019). Distributed estimation based on multi-hop subspace decomposition. Automatica, 99, 213-220.

del Nozal, A., Orihuela, L., \& Millán, P. (2017). Distributed consensus-based Kalman filtering considering subspace decomposition. IFAC-PapersOnLine, 50 (1), 2494-2499.

Dong, H., Wang, Z., Alsaadi, F., \& Ahmad, B. (2015). Event-triggered robust distributed state estimation for sensor networks with state-dependent noises. International Journal of General Systems, 44 (2), 254-266.

Dong, R., \& Geng, Z. (2016). Formation tracking control of multi-vehicle systems. Asian Journal of Control, 18(1), 350-356. 
Dong, X., Yu, B., Shi, Z., \& Zhong, Y. (2015). Time-varying formation control for unmanned aerial vehicles: theories and applications. Control Systems Technology, IEEE Transactions on, 23(1), 340-348.

Forti, N., Battistelli, G., Chisci, L., Li, S., Wang, B., \& Sinopoli, B. (2018). Distributed joint attack detection and secure state estimation. IEEE Transactions on Signal and Information Processing over Networks, 4(1), 96-110.

García, R., Rubio, F., Orihuela, L., Millán, P., \& Ortega, M. (2017). Observadores distribuidos garantistas para sistemas en red. Revista Iberoamericana de Automática e Informática Industrial RIAI, $14(3), 256-267$.

Garin, F., \& Schenato, L. (2010). A survey on distributed estimation and control applications using linear consensus algorithms. In Networked control systems (pp. 75-107). Springer.

Grilo, A., Casaca, A., Pereira, P., Buttyan, L., Goncalves, J., \& Fortunato, C. (2012, June). A wireless sensor and actuator network for improving the electrical power grid dependability. In 8th euro-nf conference on next generation internet (p. 71-78). Karlskrona, Sweden.

Keiffer, M. (2009). Distributed bounded-error state estimation. Proceedings of the International Symposium on System Identification, 360-365.

Lafferriere, G., Williams, A., Caughman, J., \& Veerman, J. (2005). Decentralized control of vehicle formations. Systems \& control letters, 54(9), 899-910.

Lawton, J., Beard, R., \& Young, B. (2003). A decentralized approach to formation maneuvers. IEEE transactions on robotics and automation, 19(6), 933-941.

Li, H., Shi, Y., \& Yan, W. (2016). Distributed receding horizon control of constrained nonlinear vehicle formations with guaranteed $\gamma$-gain stability. Automatica, 68, 148-154.

Li, H., Xie, P., \& Yan, W. (2016). Receding horizon formation tracking control of constrained underactuated autonomous underwater vehicles. IEEE Transactions on Industrial Electronics, 64(6), 5004-5013.

Lin, F., Fardad, M., \& Jovanović, M. R. (2012). Optimal control of vehicular formations with nearest neighbor interactions. Automatic Control, IEEE Transactions on, 57(9), 22032218.

Lin, M., Wu, Y., \& Wassell, I. (2008, January). Wireless sensor network: Water distribution monitoring system. In Ieee radio and wireless symposium (p. 775-778). Orlando, FL, USA.

Liu, Y., \& Bucknall, R. (2015). Path planning algorithm for unmanned surface vehicle formations in a practical maritime environment. Ocean Engineering, 97, 126-144.

Ma, L., Wang, Z., Lam, H., \& Kyriakoulis, N. (2017, Nov). Distributed event-based setmembership filtering for a class of nonlinear systems with sensor saturations over sensor networks. IEEE Transactions on Cybernetics, $47(11), 3772-3783$.

Matei, I., \& Baras, J. (2012). Consensus-based linear distributed filtering. Automatica, 48(8), $1776-1782$.

Mazenc, F., \& Bernard, O. (2011). Interval observers for linear time-invariant systems with disturbances. Automatica, 47(1), 140-147.

Millan, P., Orihuela, L., Jurado, I., \& Rodriguez Rubio, F. (2014). Formation control of autonomous underwater vehicles subject to communication delays. Control Systems Technology, IEEE Transactions on, 22(2), 770-777.

Millán, P., Orihuela, L., Vivas, C., Rubio, F. R., Dimarogonas, D. V., \& Johansson, K. H. (2013). Sensor-network-based robust distributed control and estimation. Control Engineering Practice, 21(9), 1238-1249.

Mitra, A., \& Sundaram, S. (2018). Distributed observers for LTI systems. IEEE Transactions on Automatic Control.

Olfati-Saber, R. (2007, December). Distributed Kalman filtering for sensor networks. In 46th ieee conference on decision and control (p. 5492-5498). New Orleans, LA, USA.

Orihuela, L., Millán, P., Roshany-Yamchi, S., \& Garcia, R. (2018). Negotiated distributed estimation with guaranteed performance for bandwidth-limited situations. Automatica, 87, 94-102.

Orihuela, L., Millán, P., Vivas, C., \& Rubio, F. (2016). Suboptimal distributed control and estimation: application to a four coupled tanks system. International Journal of Systems 
Science, 47(8), 1755-1771.

Orihuela, L., Roshany-Yamchi, S., Garcia, R., \& Millán, P. (2017). Distributed set-membership observers for interconnected multi-rate systems. Automatica, 85, 221-226.

Ousingsawat, J., \& Campbell, M. E. (2004). On-line estimation and path planning for multiple vehicles in an uncertain environment. International Journal of Robust and Nonlinear Control, 14(8), 741-766.

Peng, Z., Wang, D., Chen, Z., Hu, X., \& Lan, W. (2013). Adaptive dynamic surface control for formations of autonomous surface vehicles with uncertain dynamics. Control Systems Technology, IEEE Transactions on, 21(2), 513-520.

Peng, Z., Wen, G., Rahmani, A., \& Yu, Y. (2015). Distributed consensus-based formation control for multiple nonholonomic mobile robots with a specified reference trajectory. International Journal of Systems Science, 46(8), 1447-1457.

Rubio, F. R., Millán, P., Orihuela, L., \& Vivas, C. (2014). Distributed estimation and control systems over communication networks. Revista Iberoamericana de Automática e Informática Industrial $\{R I A I\}, 11(4), 377-388$.

Stoianov, I., Nachman, L., \& Madden, S. (2007, April). PIPENET: A wireless sensor network for pipeline monitoring. 6th International Symposium on Information Processing in Sensor Networks, 264-273.

Tubaishat, M., Shang, Y., \& Shi, H. (2007, Jan). Adaptive traffic light control with wireless sensor networks. In Consumer communications and networking conference, 200\%. ccnc $200 \%$. 4th ieee (p. 187-191).

Wang, G., Li, N., \& Zhang, Y. (2017). Diffusion distributed Kalman filter over sensor networks without exchanging raw measurements. Signal Processing, 132, 1-7.

Wang, Y., Alamo, T., Puig, V., \& Cembrano, G. (2018). A distributed set-membership approach based on zonotopes for interconnected systems. In 2018 ieee conference on decision and control (cdc) (pp. 668-673).

Wei, G., Liu, S., Song, Y., \& Liu, Y. (2015). Probability-guaranteed set-membership filtering for systems with incomplete measurements. Automatica, 60, 12 - 16.

Xue, D., Wu, Z., \& Wang, S. (2015). Dynamical analysis of autonomous underwater glider formation with environmental uncertainties. Procedia IUTAM, 13, 108-117.

Yang, A., Naeem, W., Irwin, G. W., \& Li, K. (2014). Stability analysis and implementation of a decentralized formation control strategy for unmanned vehicles. Control Systems Technology, IEEE Transactions on, 22(2), 706-720.

Yu-liang, T., Yu, L., Lian-fen, H., Jian, G., \& Ying, L. (2012, July). Wireless sensor network for on-line structural health monitoring. In Computer science education (iccse), 2012 7th international conference on (p. 386-389).

Zhang, X., Hengster-Movric, K., Sebek, M., Desmet, W., \& Faria, C. (2017). Distributed observer and controller design for spatially distributed systems. IEEE Transactions on Control Systems Technology(99), 1-13.

Zhou, B., Qian, K., Fang, F., Ma, X., \& Dai, X. (2015, June). Multi-sensor fusion robust localization for indoor mobile robots based on a set-membership estimator. In Cyber technology in automation, control, and intelligent systems (cyber), 2015 ieee international conference on (p. 157-162). 